\title{
Data-Assisted Low Complexity Compressive Spectrum Sensing on Real-Time Signals under Sub-Nyquist Rate
}

\author{
Zhijin Qin, Student Member, IEEE, Yue Gao, Senior Member, IEEE, and Clive G. Parini, Member, IEEE
}

\begin{abstract}
In this paper, we consider a hybrid framework combining compressive spectrum sensing with geo-location database to find the spectrum holes in cognitive radio. In the hybrid framework, a geo-location database algorithm is proposed to be stored locally at secondary users (SUs) to remove the extra transmission link to a centralized remote geo-location database. Specifically, by utilizing the output of geo-location database algorithm, a data-assisted non-iteratively reweighted least squares (DNRLS) based compressive spectrum sensing algorithm is proposed to improve detection performance under sub-Nyquist sampling rates for wideband spectrum sensing, and to reduce the computational complexity during signal recovery. In addition, an efficient method for the calculation of maximum allowable equivalent isotropic radiated power in TV white space (TVWS) is also designed to further relax SUs. The convergence and complexity of the proposed DNRLS algorithm are analyzed theoretically. Furthermore, the proposed framework is tested on real-time signals and data after having been validated by simulated signals and data in TVWS.
\end{abstract}

Index Terms-Compressive spectrum sensing, geo-location database, wideband spectrum sensing, TV white space.

\section{INTRODUCTION}

W ITH the rapid development of wireless communications, the scarcity of spectrum resources becomes an urgent problem. However, as reported by the Federal Communications Commission (FCC) and the UK Office of Communications (Ofcom), a large percentage of spectrum resources are underutilized [1], [2]. Cognitive radio (CR) is a new intelligent wireless communication technology proposed to solve the inefficiency of the fixed spectrum assignment policy [3]. Furthermore, it is noted that spectrum used to be allocated to analog TV signals has been cleaned and opened to access due to the digital-switch-over. To implement CR in TV white space (TVWS) successfully, there are mainly two goals to be achieved: 1) protect incumbent licensed users from harmful interference; 2) utilize the available spectrum efficiently [4].

In order to avoid any harmful interference to primary services in TVWS, secondary users (SUs), also named as white space devices (WSDs), should have the knowledge of spectrum occupancy. Two approaches have been proposed to make SUs aware of the spectrum occupancy. One approach is geo-location database which is a centralized database to output the maximum allowable equivalent isotropic radiated power

The authors are with Queen Mary University of London, School of Electronic Engineering and Computer Science, London, United Kingdom. (email:\{z.qin; yue.gao; c.g.parini\}@qmul.ac.uk)
(EIRP) for each vacant TVWS channel for a specific location and time [1]. So far, several geo-location database providers such as Spectrum Bridge, Nominet, Google etc. [5] have been approved by Ofcom. Geo-location database typically calculates the interference generated in wireless communication systems through theoretical propagation models rather than actual measurements, which may result in inaccurate results for spectrum occupancy [6]. Furthermore, geo-location database approach can only protect the registered users. However, some SUs may not be registered, which may pose significant challenges to a geo-location database. For example, Public Making and Special Events (PMSE) devices such as wireless microphone operate mostly on an unlicensed basis, without any record in TVWS [7]. Therefore, the approach to protect unregistered applications is spectrum sensing. Spectrum sensing requires SUs to have the capability to detect spectrum holes that are not occupied by primary users (PUs). This approach provides instant channel occupancy information, but it may cause interference to some reserved channels which would be determined as vacant by sensing only. Therefore, a geo-location database can be utilized to improve the accuracy of spectrum sensing.

So far, some work has been researched on the combination of spectrum sensing and geo-location database. Wang et al. [8] proposed a hybrid framework combining spectrum sensing with geo-location database was proposed, in which the utilization of spatial-temporal spectrum hole is maximized. Wang and Gao et al. [9] proposed to combine the advantages of spectrum sensing and geo-location database, in which different spectrum sensing modules are performed based on the output of geo-location database. Qin et al. [10] proposed a compressive spectrum sensing algorithm to improve the sensing performance by utilizing the information provided by geo-location database. Furthermore, Ribeiro et al. [7] implemented a hybrid framework into an experimental platform by combining wireless microphone sensors with a web-based geo-location database access for PMSE. However, all the existing frameworks required that SUs should build a direct link to the remote geo-location database. This direct link causes increasing loads in CR networks and transmission errors between the SUs and remote geo-location database.

Recently, wideband spectrum sensing has attracted much attention. As required by the Nyquist sampling theory, a simple approach to wideband spectrum sensing is to directly acquire the wideband signal by a high-speed analog-to-digital converter (ADC). For example, Quan et al. [11] proposed 
a multi-band joint detection algorithm to sense PUs over wideband spectrum by using a high-speed ADC for signal acquisition. Furthermore, Tian and Giannakis [12] proposed a wavelet-based wideband spectrum sensing algorithm by implementing a high-speed ADC. However, the high-speed ADC is particularly challenging for power-limited devices such as smart phones, slave WSDs or even battery-free devices in a wireless power transfer model [13]. Lately, Landau [14] demonstrated that sampling rate should be no less than the measure of occupied part of the spectrum for the stable reconstruction of multiband signals. Furthermore, the spectrum exhibits a sparse property in the frequency domain as its low utilization in practice [1], [2]. Compressive sensing (CS) was proposed recently to reduce the sampling rate under subNyquist rate by utilizing this sparse property of spectrum [15].

CS was firstly applied to spectrum sensing by Tian and Giannakis [16], where fewer compressed measurements are required on the basis of Nyquist sampling theory. Subsequently, the CS based wideband spectrum sensing has attracted much attention [17]-[19]. Wang et al. [17] proposed a twostep CS scheme for minimizing the sampling rates when the sparsity level is changing. In this approach, the actual sparsity level is estimated firstly and the number of compressed measurements to be sampled are then adjusted before sampling. In addition, Sun et al. [18] proposed to adjust the number of compressed measurements adaptively without sparsity estimation by acquiring compressed samples step by step in continuous sensing slots. Signal acquisition is terminated once the number of collected samples were enough for successful spectral recovery. In addition, Qin et al. [19] proposed a denoised $l_{1}$ minimization based spectrum sensing algorithm for the machine-to-machine (M2M) communication network. However, for the implementation of compressive spectrum sensing, innovative technologies are required to continuoustime signal acquisition. Mishali and Eldar [20] proposed a modulated wideband converter model to provides the robustness against the noise and model mismatches. Tropp et al. [21] proposed an analog-to-information converter (AIC) sampler to make the analog CS implementable. The AIC sampler has attracted much attention for practical implementation and it has been adopted as the measurement matrix in [17].

Many of the aforementioned compressive spectrum sensing algorithms utilize $l_{1}$ minimization. However, as pointed out in [22], large coefficients are penalized more heavily than smaller coefficients in $l_{1}$ minimization, which may lead to performance degeneration. In order to rectify a key difference between $l_{0}$ and $l_{1}$ minimization and balance the penalty on large coefficients and smaller coefficients of the sparse signal, Candes et al. [22] proposed an iteratively reweighted $l_{1}$ minimization algorithm by introducing weight for each bin of the signal to be recovered. Another approach to recover a sparse signal with fewer measurements is to replace the $l_{1}$ norm with $l_{p}$ norm. In order to solve the $l_{p}$ norm problem, an iteratively reweighted least squares (IRLS) algorithm was proposed to perform sparse signal reconstruction [23]-[28].

So far, recovering compressed samples by utilizing prior information has been studied in [29]-[32]. Oscar et al. [29] proposed the prior information assisted sparse signal ap- proximation algorithms: weighted basis pursuit denoising and weighted match pursuit. In addition, two partial support information assisted CS algorithms were proposed respectively in [30] and [31], in which the weighted $l_{1}$ minimization approach with fixed weights on the known support is utilized to find the sparse solution for CS problems. Furthermore, Miosso et al. [32] proposed an IRLS based CS recovery algorithm utilizing the prior information, in which the weights are updated in each iteration of the IRLS algorithm. The different iterative approaches for weight setting in IRLS were compared in [33]. However, the iterative weight updating approach in IRLS introduces extra computational complexities for signal recovery.

Motivated by the challenges identified above, the main contributions of this paper are listed as follows:

1) A hybrid framework combining compressive spectrum sensing with geo-location database is proposed in which a geo-location database algorithm is implemented at SUs locally to provide prior information for the compressive spectrum sensing.

2) In the proposed framework, a data-assisted noniteratively reweighted least squares (DNRLS) based compressive spectrum sensing algorithm with lower computational complexity and lower necessary subNyquist sampling rate is proposed. In the proposed DNRLS, the data generated by the locally stored geolocation database algorithm at SUs is utilized to replace the iterative process of weights updating in IRLS algorithm. Convergence and computational complexity of the proposed DNRLS are analysed.

3) In addition, an efficient approach for calculating the maximum allowable EIRP is proposed to further improve the accuracy and efficiency of the geo-location database algorithm stored at SUs.

4) Furthermore, based on the recent work on the trail within the Ofcom TVWS pilot [34], the proposed framework and algorithms are tested on real-time signals and data recorded by the CRFS RFeye node [35] and the regulator qualified geo-location database from Nominet [36] after having been validated by the simulated signals and data.

The rest of this paper is organized as follows. Section II describes the system models for compressive spectrum sensing and geo-location database. Section III presents the proposed hybrid framework with the DNRLS based compressive spectrum sensing algorithm and the Wilkinsons method based geolocation database calculation algorithm. The numerical analyses for the proposed algorithms are illustrated in section IV. The conclusions are drawn in section $\mathrm{V}$.

\section{SySTEM MODEL}

In this section, models are introduced for both compressive spectrum sensing and geo-location database.

\section{A. Compressive spectrum sensing model}

The compressive spectrum sensing model contains four components: sparse representations of received signals, compressed measurements collection, signal recovery and spec- 
trum sensing decision making. In this model, the received signal at a $\mathrm{SU}$ is $r(t)=h(t) * s(t)+n(t)$, where $s(t) \in C^{N \times 1}$ is the sum of multiple primary signals in the spectrum of interest. In addition, $h(t)$ is the channel gain between the transmitters and receivers, and $n(t) \sim \mathcal{C N}\left(0, \sigma^{2} \mathbf{I}_{N}\right)$ refers to Additive White Gaussian Noise (AWGN) with zero mean and variance $\sigma^{2}$.

Representations of the received signal $r(t)$ in the frequency domain can be expressed as

$$
r_{f}=h_{f} s_{f}+n_{f},
$$

where $r_{f}, h_{f}, s_{f}$ and $n_{f}$ are the discrete Fourier transforms (DFT) of $r(t), h(t), s(t)$ and $n(t)$. The transmitted signal $s_{f}$ exploits a sparse property in frequency domain as spectrum is normally underutilized in practice. This sparse property makes it possible to reduce sampling rates by implementing CS at SUs.

When CS is applied at a SU, the collected compressed measurements can be expressed as

$$
x=\Phi \mathcal{F}^{-1} r_{f}=\Theta r_{f}=\Theta\left(h_{f} s_{f}+n_{f}\right),
$$

where $\Phi \in C^{P \times N}(P \leq N)$ is a measurement matrix. It is utilized to collect the compressed measurements, $x \in C^{P \times 1}$, with $P / N \leq 1$ being the compression ratio. The measurement matrix can be a matrix which contains a single spike in each row. The case $P / N=1$ corresponds to $\Phi=\mathbf{I}_{N}$. In addition, $\Theta=\Phi \mathcal{F}^{-1}$, where $\mathcal{F}^{-1}$ is inverse DFT matrix which is used as the sparsifying matrix.

In practical settings, structured random matrices are often employed for improved implementation affordability. In this paper, we take AIC sampler from [21] to realize the subNyquist sampling. The AIC sampler contains three components: a high-rate pseudonoise sequence, a low-pass antialising filter and a low speed ADC. We let $\left(\varepsilon_{0}, \varepsilon_{1}, \cdots, \varepsilon_{N-1}\right)$ be the chipping sequences, and demodulation matrix corresponds to a $N \times N$ diagonal matrix, where $D=$ $\operatorname{diag}\left\{\varepsilon_{0}, \cdots, \varepsilon_{N-1}\right\}$. Action of the accumulate-and-dump sampler is given by matrix $H$ of size $P \times N$ whose $p$ th row has $P / N$ consecutive unit entries starting in column $p N / P+1$ for each $p=0,1, \ldots, P-1$. In summary, the matrix $\Theta=H \cdot D \cdot \mathcal{F}^{-1}$ describes the overall action of the hardware system on signal $r_{f}$. This structure alleviates the burden on the ADC, at the expense of slightly degraded recovery performance compared with those fully random Gaussian sampler. As shown in [37], there are three conditions for $\Theta: 1)$ each column of $\Theta$ is normalized, 2) each row has approximately equal norm, and 3) the rows of $\Theta$ are orthogonal. These three conditions can be fulfilled by random matrices such as the AIC sampler.

After the compressed measurements are collected at subNyquist sampling rates by the AIC sampler, the original signals should be reconstructed before making decision on spectrum occupancy. Signal recovery can be formulated as a convex optimization problem and solved by $l_{1}$ minimization as

$$
\begin{aligned}
& \min \left\|\hat{s}_{f}\right\|_{1} \\
& \text { subject to }\left\|\Theta \cdot h_{f} \hat{s}_{f}-x\right\|_{2}^{2} \leq \eta,
\end{aligned}
$$

where $\eta$ is the error bound related to the noise level.

A non-convex variant of the $l_{1}$ minimization can produce exact reconstruction with fewer measurements [23]. Specifically, the $l_{1}$ norm is replaced by the $l_{p}$ norm, where $0 \leq p \leq 1$. Therefore, (3) can be expressed as

$$
\begin{aligned}
& \min \left\|\hat{s}_{f}\right\|_{p} \\
& \text { subject to }\left\|\Theta \cdot h_{f} \hat{s}_{f}-x\right\|_{2}^{2} \leq \eta
\end{aligned}
$$

When the recovered signal $\hat{s}_{f}$ is obtained by solving the above optimization problem, energy detection is performed to determine the spectrum occupancy by comparing the energy of recovered signal with a predefined threshold defined as follows [38]:

$$
\lambda_{d}=\sigma^{2}\left(1+\frac{Q^{-1}\left(P_{f}\right)}{\sqrt{N / 2}}\right) .
$$

In (5), $\sigma^{2}$ is the noise variance, and $P_{f}$ refers to the target probability of false alarm of spectrum sensing, and $N$ is the number of samples used for decision making in energy detection. In practice, the noise variance is "a prior" and needs to be estimated before making decision on spectrum occupancy. Threshold learning [39], [40] is typically used in conjunction with noise estimation [41] in order to make the CR system be really cognitive. After noise level is estimated, the energy density of each considered channel should be compared with the threshold. If greater than the threshold, the corresponding channel is determined as occupied by PUs, and SUs are forbidden to access it. Otherwise, the corresponding channel is determined as vacant, and the SUs can access it to transmit the unlicensed signals.

\section{B. Geo-location database model}

For geo-location databases a power control model is currently used to calculate the maximum allowable EIRP in [42]. A two-ray path loss model is used to measure the power attenuation. However, there are two main problems for this model. Firstly, the digital terrestrial television (DTT) receivers can not be located precisely. This infers that the path loss between SUs and DTT receivers can not be calculated accurately. Secondly, different environmental scenarios that would lead to different tolerance levels for DTT receivers, are not considered in the power control model. For example, the power attenuation in urban areas is much higher than open areas. As a result, the maximum allowable EIRP of a specific SU in open areas is greater than that in urban areas.

To solve these problems, the location probability model introduces the concept of location uncertainty, which classifies the location relationship between SUs and DTT receivers into four different scenarios. In addition, transmission environment can be classified as open, suburban and urban areas. Both the location relationship and transmission environment between SUs and DTT receivers would influence the power attenuation. DTT location probability is defined as the probability with which a DTT receiver could operate accurately at a specific location; i.e., the probability with which the average received wanted signal level is appropriately greater than a minimum required value. DTT location probability is used to limit the 
maximum allowable EIRP $P_{I B}$ and it can be expressed in linear domain as follows [43]:

$$
\begin{aligned}
& q_{1}=\operatorname{Pr}\left\{P_{s} \geq P_{s, \min }+\sum_{k=1}^{K} r_{U, k} P_{U, k}\right\}, \\
& q_{2}= \\
& \operatorname{Pr}\left\{P_{s} \geq P_{s, \min }+\sum_{k=1}^{K} r_{U, k} P_{U, k}+r\left(\Delta f, m_{s}\right) G P_{I B}\right\},
\end{aligned}
$$

where $P_{s}$ is the average received power of wanted DTT signal and $P_{s(d B m)}$ is modeled as a Gaussian random variable with mean $m_{s}$ and standard deviation $\sigma_{s}$. In addition, $P_{s, \min }$ is DTT receiver's reference sensitivity level. The parameter $P_{U, k}$ refers to received power of the $k$ th unwanted DTT signals, and $K$ is the total number of received unwanted DTT signals. Parameter $r_{U, k}$ is the protection ratio of the received wanted DTT power and received $k$ th unwanted DTT power at the point where DTT receiver fails. The difference $\Delta f=f_{\mathrm{WSD}}-f_{\mathrm{DTT}}$, where $f_{\mathrm{WSD}}$ is the frequency in which a WSD device operates and $f_{\text {DTT }}$ is the DTT carrier frequency. $G$ is defined as the power attenuation factor coupling gain. The DTT receiver's location probability in the absence of interference from WSDs is labelled as $q_{1}$, and $q_{2}$ is the DTT receiver's location probability when considering the additional interference caused by WSDs. When the interference from WSDs is considered, it results in a reduction in location probability $\Delta q=q_{1}-q_{2}$. To identify the maximum allowable EIRP $P_{I B}$ in (7), $\Delta q$ is maximized by assigning a maximal allowable value $\Delta q_{T}$ to $\Delta q$.

We can express (6) in decibel domain as follows [44]:

$$
\begin{aligned}
q_{1} & =\operatorname{Pr}\left\{P_{s} \geq P_{s, \min }+\sum_{k=1}^{K} r_{U, k} P_{U, k}\right\} \\
& =\operatorname{Pr}\left\{P_{s} \geq P_{s, \min }+V\right\} \\
& =\operatorname{Pr}\left\{1 \geq \frac{P_{s, \min }}{P_{s}}+\frac{V}{P_{s}}\right\} \\
& =\operatorname{Pr}\{1 \geq A+B\} \\
& =\operatorname{Pr}\{1 \geq X\},
\end{aligned}
$$

where $V_{(d B m)}$ is modeled as a Gaussian random variable with mean $m_{V}$ and standard deviation $\sigma_{V}$. Furthermore, $A_{(d B)}$ and $B_{(d B)}$ can be modeled as Gaussian random variables. In addition, $X_{(d B)}$ can be modeled as a Gaussian random variable with mean $m_{X}$ and standard deviation $\sigma_{X}$. As a result, (8) can be given by

$$
q_{1}=\operatorname{Pr}\left\{0 \geq X_{(d B)}\right\}=\frac{1}{2} \operatorname{erfc}\left(\frac{m_{X}}{\sqrt{2} \sigma_{X}}\right) .
$$

Similarly, $q_{2}$ can be expressed in decibel domain as follows:

$$
\begin{aligned}
& q_{2}= \\
& \begin{aligned}
\operatorname{Pr} & \left\{P_{s} \geq P_{s, \text { min }}+\sum_{k=1}^{K} r_{U, k} P_{U, k}+r\left(\Delta f, m_{s}\right) G P_{I B}\right\} \\
& =\operatorname{Pr}\left\{P_{s} \geq P_{s, \min }+V+r\left(\Delta f, m_{s}\right) G P_{I B}\right\} \\
& =\operatorname{Pr}\left\{1 \geq \frac{P_{s, \min }}{P_{s}}+\frac{V+r\left(\Delta f, m_{s}\right) G P_{I B}}{P_{s}}\right\} \\
& =\operatorname{Pr}\left\{1 \geq A+\frac{V+C}{P_{s}}\right\} \\
& =\operatorname{Pr}\left\{1 \geq A+\frac{D}{P_{s}}\right\} \\
& =\operatorname{Pr}\{1 \geq A+E\} \\
& =\operatorname{Pr}\{1 \geq Y\},
\end{aligned}
\end{aligned}
$$

where $C_{(d B m)}$ is a Gaussian random variable with mean $m_{C}$ and standard deviation $\sigma_{C} . V$ and $C$ are two uncorrelated lognormal random variables, and then $D_{(d B m)}$ can be modeled as a Gaussian random variable with mean $m_{D}$ and standard deviation $\sigma_{D}$. Furthermore, as $D$ and $P_{S}$ are both log-normal random variables, $E_{(d B)}$ is also Gaussian variable with $m_{E}=$ $m_{D}-m_{S}$ and $\sigma_{E}=\sqrt{\sigma_{D}^{2}+\sigma_{S}^{2}}$. Eventually, as $A$ and $\mathrm{E}$ are both log-normal random variables, $Y_{(d B)}$ can be modeled as a Gaussian random variable with mean $m_{Y}$ and standard deviation $\sigma_{Y}$. Furthermore, (10) can be expressed as

$$
q_{2}=\operatorname{Pr}\left\{0 \geq Y_{(d B)}\right\}=\frac{1}{2} \operatorname{erfc}\left(\frac{m_{Y}}{\sqrt{2} \sigma_{Y}}\right) .
$$

Once $q_{2}$ is obtained, the corresponding $P_{I B}$ can be calculated for each channel to indicate the maximum allowable EIRP as the output of the geo-location database.

\section{The Proposed Hybrid Framework Combining Compressive Spectrum SENSING With GeO-LOCATION DATABASE}

In the wideband spectrum sensing scenario, as shown in Fig. 1 (a), multiple PUs exist in the multiband spectrum of interest and each SU should be capable to detect the active PUs accurately and efficiently. The traditional hybrid frameworks with geo-location database and spectrum sensing proposed in [7]-[9] require a direct link to the remote geo-location database as shown in Fig. 1 (b). Dynamic changes of the spectrum would not be reflected unless it is registered and updated in the centralized geo-location database. This process introduces several information exchanges such as the two-way transmissions between the SU and the geo-location database. In addition, each transmission link introduces potential errors as well as energy consumption.

In order to reduce the necessary sampling rates at SUs and alleviate both the network load and the inevitable transmission errors between geo-location database and SUs, we propose a hybrid framework compressive spectrum sensing with geo-location database algorithm as shown in Fig. 1 (c). In the proposed hybrid framework, a geo-location database calculation algorithm is stored at SUs locally. Meanwhile, the DTT transmitter location information is maintained at the corresponding $\mathrm{SU}$, which is used for the geo-location database calculation algorithm. The maximum allowable EIRP $P_{I B}$ of each channel obtained from geo-location database algorithm is fused with the historical $P_{I B}$ and utilized as the prior information for the compressive spectrum sensing. As a result, the necessary sampling rates for exact signal recovery and computational complexities are reduced at SUs. In addition, in order to further relax the SU, a Wilkinson's method [45] based DTT location probability is proposed to calculate the maximum allowable EIRP $P_{I B}$ of each channel efficiently and locally.

\section{A. The proposed data-assisted non-iteratively reweighted least squares based compressive spectrum sensing}

Before introducing the proposed DNRLS based compressive spectrum sensing algorithm, we firstly introduce the IRLS algorithm. When IRLS algorithm is utilized to solve (4), the 


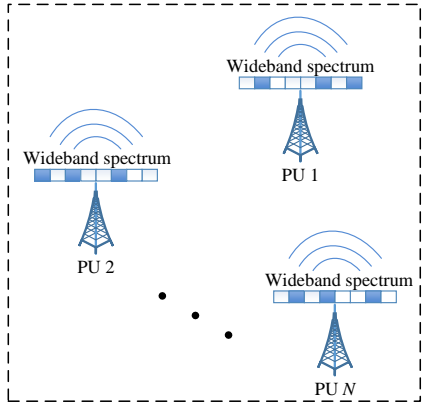

(a) Multiple primary users (PUs) with wideband spectrum

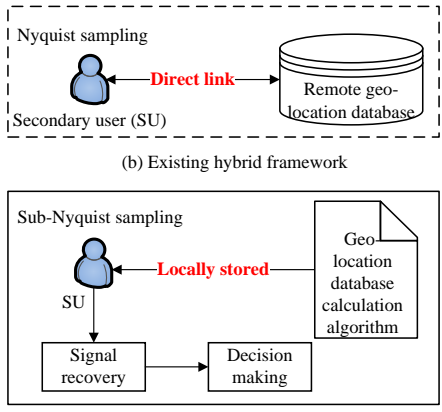

(c) The proposed hybrid framework
Fig. 1: (a). Scenario of wideband spectrum sensing with multiple primary users (PUs); (b). the existing hybrid framework with a direct link to remote database and conventional spectrum sensing at Nyquist rate; and (c). the proposed hybrid framework with the locally stored geo-location database calculation algorithm and the data-assisted non-iteratively reweighted least squares (DNRLS) based compressive spectrum sensing.

optimization problem can be formulated in Lagrangian form as follows:

$$
\min \left\|\Theta \cdot h_{f} \hat{s}_{f}-x\right\|_{2}^{2}+\lambda W \cdot \hat{s}_{f}^{2},
$$

where $W$ is a diagonal matrix $W=$ $\operatorname{diag}\left\{w_{1}, \ldots, w_{n}, \ldots, w_{N}\right\}$ which is updated in each iteration. In the $l$ th $(l=0,1, \ldots, L)$ iteration of IRLS algorithm, the weights are calculated with the recovered signal $\hat{s}_{f_{-} n}^{(l-1)}$ in the $(l-1)$ th iteration as

$$
w_{n}^{(l)}=\left(\left(\hat{s}_{f_{-} n}^{(l-1)}\right)^{2}+\varepsilon^{(l)}\right)^{1-\frac{p}{2}} .
$$

In (13), $\varepsilon^{(l)}$ is updated in each iteration, and it is a positive value to make sure that a zero-valued component in $\bar{s}_{f}$ does not strictly prohibit a non-zero estimate in the next iteration of weights update. In addition, the solution of (12) at the $l$ th iteration can be expressed as

$$
\begin{aligned}
\hat{s}_{f_{-} n}^{(l)} & =\arg \min \left\|\Theta \cdot h_{f} \hat{s}_{f}-x\right\|_{2}^{2}+\lambda W^{(l)} \cdot \hat{s}_{f}^{2} \\
& =W^{(l)} \Theta^{\mathrm{T}}\left(h_{f} \Theta W^{(l)} \Theta^{\mathrm{T}}+\lambda \mathbf{I}_{P}\right)^{-1} x,
\end{aligned}
$$

where the initial value for the weights $w_{n}$ in $W$ is 1 , and then $W^{(0)}=\mathbf{I}_{N}$. As a result, $\hat{s}_{f_{-} n}^{(0)}=\Theta^{\mathrm{T}}\left(h_{f}+\lambda \mathbf{I}_{P}\right)^{-1} x$. It is noted that (12) is a non-convex optimization problem when $p<1$, and as such, the solution to (12) can be local minima. It has been shown that the computed local minimizers of (12) are global minimizer when solved by IRLS [23]-[25].

Definition 1: The restricted isometry property (RIP) guarantees the stable and robust recovery by solving the optimization problem (4). We say that a matrix $\Theta$ satisfies the property $P(a, \tilde{K}, p)$ if it satisfies

$$
\delta_{a \tilde{K}}+a^{\frac{2}{p}-1} \delta_{(a+1) \tilde{K}}<a^{\frac{2}{p}-1}-1,
$$

where $a>1$, and $\tilde{K}$ is sparsity level of the spectrum of interest.
Theorem 1 [27]: Let $0<p \leq 1$. If a $P \times N$ matrix satisfies $P(a, \tilde{K}, p)$, then

$$
\left\|\hat{s}_{f}-s_{f}\right\|_{2}^{p} \leq C^{1} \eta+C^{2} \frac{\left\|s_{f}-s_{f, \tilde{K}}\right\|_{p}^{p}}{\tilde{K}^{\frac{2}{p}-1}},
$$

where

$$
\begin{gathered}
C^{1}=2^{p} \frac{1+a^{\left(\frac{p}{2}-1\right)}\left(\frac{p}{2}-1\right)^{-\frac{p}{2}}}{\left(1-\delta_{(a+1) \tilde{K}}\right)^{\frac{p}{2}}-\left(1+\delta_{a \tilde{K}}\right)^{\frac{p}{2}} a^{\left(\frac{p}{2}-1\right)}}, \text { and } \\
C^{2}=\frac{2\left(\frac{p}{2-p}\right)^{\frac{p}{2}}}{a^{\left(1-\frac{p}{2}\right)}}\left(1+\frac{\left(\left(\frac{p}{2}-1\right)^{-\frac{p}{2}}+a^{\left(\frac{p}{2}-1\right)}\right)\left(1+\delta_{a \tilde{K}}\right)^{\frac{p}{2}}}{\left(1-\delta_{(a+1) \tilde{K}}\right)^{\frac{p}{2}}-\left(1+\delta_{a \tilde{K}}\right)^{\frac{p}{2}} a^{\left(\frac{p}{2}-1\right)}}\right) .
\end{gathered}
$$

In the traditional IRLS based compressive sensing in (12), the key challenge is to find the optimal set of weights $W$ in an iterative process for a better estimate of the original signals. It should be noted the iterations generate more computational complexities during signal recovery process. When part of the maximum allowable EIRP is available in advance, the iterative process can be removed without degrading the recovery performance heavily. In this paper, we propose a DNRLS based compressive spectrum sensing algorithm. In the proposed algorithm, a geo-location database algorithm is implemented at SUs locally to provide data for weights calculation. This is achieved by a non-iterative method so that SUs would not need any additional link to a centralized geolocation database. The proposed calculation yields the weights as

$$
w_{n}=\left(\left|\bar{\gamma}_{n}\right|^{2}+\varepsilon\right)^{1-\frac{p}{2}},
$$

where $\varepsilon$ is a positive value same as $\varepsilon^{(l)}$ in (13), and $\bar{\gamma}=$ $\left\{\bar{\gamma}_{1}, \ldots \bar{\gamma}_{n}, \ldots \bar{\gamma}_{N}\right\}$ is constructed by the channel historical data and the output of geo-location database algorithm. The construction of $\bar{\gamma}$ in detail is introduced in the following.

In the $(t+1)$ th sensing period, the maximum allowable EIRP $P_{I B}(t+1)$ is calculated for the current period by the proposed Wilkinson's method based DTT location probability calculation algorithm introduced in section III-B. Subsequently, the $P_{I B}(t+1)$ is mapped to $\gamma(t+1)$. Furthermore, the averaged $\bar{\gamma}(t+1)$ is calculated as

$$
\bar{\gamma}(t+1)=(\bar{\gamma}(t)+\gamma(t+1)) / 2,
$$

where $\bar{\gamma}(t)$ is the historical data for the weights construction at the $t$ th sensing period, where $t=\{0,1, \ldots, T\}$. Herein $T$ is the window size for SUs to fuse the current allowable maximum $P_{I B}$ with the historical data. At a SU, only the $\bar{\gamma}(t)$ is stored locally after the $t$ th sensing period. If there is any new unregistered user showing up in the spectrum of interest in $t$ th period, the related DTT transmitter information used for geo-location database calculation algorithm is updated locally. This makes the proposed weights calculation robust to the new unregistered users. Meanwhile, the geo-location database at other SUs would not be influenced. In the $(t+1)$ th period, the $\gamma(t+1)$ provided by the local geo-location database would be updated accordingly by considering the unregistered users. 
After $\bar{\gamma}(t+1)$ for the current sensing period is obtained to calculate the weights, a more accurate spectrum estimation can be obtained by solving the following non-iterative problem

$$
\begin{aligned}
\hat{s}_{f} & =\arg \min \left\|\Theta \cdot h_{f} \hat{s}_{f}-x\right\|_{2}^{2}+\lambda \tilde{W} \cdot \hat{s}_{f}^{2} \\
& =\tilde{W} \Theta^{\mathrm{T}}\left(h_{f} \hat{s}_{f} \tilde{W} \Theta^{\mathrm{T}}+\lambda \mathrm{I}_{P}\right)^{-1} x .
\end{aligned}
$$

In (19), $\tilde{W}=\operatorname{diag}\left(w_{1}, \ldots, w_{n}, \ldots, w_{N}\right)$ is a diagonal matrix in which $w_{n}$ is calculated by (17) to replace the iterative update process in (13). In the proposed DNRLS based compressive spectrum sensing algorithm, the accuracy of $\bar{\gamma}$ would influence the recovery performance.

1) Convergence: If there is no unregistered user in the spectrum of interest, which means the values of $\gamma$ used to construct the weights are accurate, the recovery performance of DNRLS is very good. When the unregistered users show up in the spectrum of interest at the 1st sensing period, the $\bar{\gamma}(1)$ becomes inaccurate on the corresponding bins as the output of the local geo-location database algorithm $\gamma(1)$ for the 1 st period is inaccurate. As a result, the signal recovery and detection performance would be degraded accordingly. In the $t$ th period after the unregistered user shows up in the spectrum of interest, $\gamma(t)$ is fused with the historical data $\bar{\gamma}(t-1)$ of the $(t-1)$ th period. The accuracy of weights $\bar{\gamma}(T)$ are dependent on the window size $T$ for the weights fusion at SUs. The weights fusion process is shown as follows:

$$
\begin{array}{cc}
\bar{\gamma}(1)=\frac{\bar{\gamma}(0)+\gamma(1)}{2}, & (\text { 1st period }) \\
\bar{\gamma}(2)=\frac{\bar{\gamma}(1)+\gamma(2)}{2}, & (\text { 2nd period }) \\
\vdots & \\
\bar{\gamma}(T)=\frac{\bar{\gamma}(T-1)+\gamma(T)}{2}, & \text { (Tth period) }
\end{array}
$$

where $\bar{\gamma}(0)$ is the historical data for weights construction before unregistered user showing up, and $\gamma(1)$ is the output of the locally implemented geo-location database algorithm for the period when unregistered users show up in the spectrum of interest. As $\gamma(2)=\cdots=\gamma(T)=\gamma$, which represents the real spectrum status with consideration of the unregistered users in the spectrum of interest, $\bar{\gamma}(T)$ can be expressed as

$$
\begin{aligned}
\bar{\gamma}(T) & =\frac{\bar{\gamma}(0)}{2^{T}}+\frac{\gamma(1)}{2^{T}}+\frac{\frac{1}{2} \times\left(1-\left(\frac{1}{2}\right)^{T-1}\right) \times \gamma}{1-\frac{1}{2}} \\
& =\frac{\bar{\gamma}(0)}{2^{T}}+\frac{\gamma(1)}{2^{T}}+\left(1-\left(\frac{1}{2}\right)^{T-1}\right) \times \gamma
\end{aligned}
$$

It is noted that $\bar{\gamma}(T)$ would converge fast to $\gamma$ after unregistered users show up in the spectrum of interest. In addition, part of channels in TVWS are fixed and utilized by DTV signals, and some of the channels are reserved for other purposes. As result, at least the weights for those fixed channels in $\bar{\gamma}(0)$ and $\gamma(1)$ are accurate. This characteristic provides a guarantee that the recovery performance would not be degraded heavily when unregistered users show up in the spectrum of interest. With increasing window size $T$, the influence of inaccurate parts in $\bar{\gamma}(0)$ and $\gamma(1)$ degrades. The influence of the window size $T$ is shown in the numerical analyses part of Section IV.
2) Complexity: The computational complexity reduction of the proposed DNRLS based compressive spectrum sensing comes from following three parts. Firstly,in the traditional IRLS algorithm, the inverse of $\left(h_{f} \Theta W^{(l)} \Theta^{T}+\lambda \mathrm{I}_{P}\right)$ takes $\mathrm{O}\left(P^{3}\right)$ and it is required in each iteration. In large size compressive sensing problem, solving a problem with complexity $\mathrm{O}\left(P^{3}\right) L$ times is unacceptable. As summarized in Algorithm 1, the proposed DNRLS based compressive sensing algorithm solves the signal recovery problem in a non-iterative approach. Therefore, the computational complexity is $1 / L$ of the traditional IRLS based compressive spectrum sensing in which $L$ iterations are required to get an accurate estimation of the spectrum. The second reason of complexity reduction is that the proposed DNRLS algorithm can achieve exact signal recovery with fewer measurements. In the proposed DNRLS algorithm, the minimal number of measurements $P$ for exact recovery is reduced to $\tilde{P}(\tilde{P}<P)$. It leads to a large computational complexity reduction as the complexity of solving the inverse of $\left(h_{f} \Theta W^{(l)} \Theta^{T}+\lambda \mathrm{I}_{P}\right)$ is $\mathrm{O}\left(\tilde{P}^{3}\right)$. The performance analyses are further shown in numerical analyses. Thirdly, the computational complexity reduction comes from the calculation of $P_{I B}$ in the proposed hybrid framework. Specifically, to minimize the necessary computational complexity at SUs, the Wilkilson's method is utilized to calculate the $P_{I B}$ for each TVWS channel. The details of the Wilkilson's method based DTT location probability calculation algorithm are introduced in section III-B.

\section{B. The proposed Wilkinson's method based DTT location probability calculation algorithm}

At a SU, the calculation of maximum allowable EIRP $P_{I B}$ of each channel in TVWS should be efficient and accurate. Monte Carlo method and Schwartz-Yeh's method are the two algorithms approved by regulators to calculate the maximum allowable EIRP $P_{I B}$. Schwartz-Yeh's method is an approximate algorithm in which infinite loops are used to calculate the mean and standard deviation of log-normal distribution variables such as variables $A, B$ and $E$ in (8) and (10) [46]. However, the large computational complexity and low efficiency of the Schwartz-Yeh's method are difficult to overcome at power-limited SUs. In this paper, we propose to use Wilkinson's method to calculate $q_{1}, q_{2}$ and $P_{I B}$ in a much more efficient way.

1) Wilkinson's method: Assuming $I_{k}(k=1,2, \ldots, K)$ is a log-normal random variable, then $M_{k}=10 \log _{10} I_{k}$ can be modeled as a Gaussian random variable and $M=$ $10 \log _{10}\left(\sum_{k=1}^{K} 10^{\frac{M_{k}}{10}}\right)$. It is assumed that $e^{\Lambda_{1}}+e^{\Lambda_{2}}+\cdots+$ $e^{\Lambda_{K}}=e^{Z}=10^{M}, Z=\rho M$, and $\rho=\frac{1}{10} \ln 10=0.2302$, the mean and standard deviation of parameter $M$ could be calculated by introducing two parameters $\mu_{1}$ and $\mu_{2}$ as follows:

$$
\mu_{1}=\mathrm{E}\left(m_{Z}+\frac{1}{2} \sigma_{Z}^{2}\right)=\sum_{i=1}^{K} \mathrm{E}\left(m_{\Lambda_{i}}+\frac{1}{2} \sigma_{\Lambda_{i}}^{2}\right)
$$


$\overline{\text { Algorithm } 1 \text { Data-assisted non-iteratively reweighted least }}$ squares based compressive spectrum sensing

1: Input: $p, \lambda, \Theta, x, \varepsilon, \bar{\gamma}(t)$.

2: Calculate $P_{I B}(t+1)$ by the proposed Wilkinson's method based DTT location probability model introduced in section III-B.

3: Map $P_{I B}(t+1)$ to $\gamma(t+1)$.

4: Calculate $\bar{\gamma}(t+1)$ by (18).

5: Perform signal recovery by (19) to get $\hat{s}_{f}$.

6: Make decision on spectrum occupancy by compare $\hat{s}_{f}$ with the threshold $\lambda_{d}$ defined in (5).

$$
\begin{aligned}
\mu_{2} & =\mathrm{E}\left(2 m_{Z}+2 \sigma_{Z}^{2}\right) \\
& =\sum_{i=1}^{K} \mathrm{E}\left(2 m_{\Lambda_{i}}+2 \sigma_{\Lambda_{i}}^{2}\right) \\
& +2 \sum_{i=1}^{K-1} \sum_{j=i+1}^{K} \mathrm{E}\left(m_{\Lambda_{i}}+m_{\Lambda_{j}}\right) \\
& \times \mathrm{E}\left[\frac{1}{2}\left(\sigma_{\Lambda_{i}}^{2}+\sigma_{\Lambda_{j}}^{2}+2 r_{i j} \sigma_{\Lambda_{i}} \sigma_{\Lambda_{j}}\right)\right],
\end{aligned}
$$

where $m_{\Lambda_{i}}$ and $\sigma_{\Lambda_{i}}$ are the mean and standard deviation of $\Lambda_{i}$, and $r_{i j}$ are the correlation coefficients of $\Lambda_{i}$ and $\Lambda_{j}$. Consequently, the mean and standard deviation of $M$ can be calculated as

$$
\begin{gathered}
\sigma_{M}=\frac{1}{\rho}\left(2 \ln \mu_{1}-\frac{1}{2} \ln \mu_{2}\right), \\
\sigma_{M}=\frac{1}{\rho}\left(\ln \mu_{2}-2 \ln \mu_{1}\right)^{\frac{1}{2}} .
\end{gathered}
$$

2) Maximum allowable equivalent isotropic radiated power calculation: Based on the Wilkinson's method explained above, $q_{1}$ and $q_{2}$ can be calculated accordingly. Taking the calculation of $q_{1}$ as an example, as shown in (8), $\frac{P_{s, \min }}{P_{s}}+\frac{V}{P_{s}}=$ $A+B \leq 1.10 \log _{10}(A+B) \leq 0$, which is equivalent to $X_{(d B)}=10 \log _{10}\left(10^{\frac{A_{d B}}{10}}+10^{\frac{B_{d B}}{10}}\right) \leq 0$. It can be fitted into the precondition of Wilkinson's method to get $10^{\frac{A_{d B}}{10}}+$ $10^{\frac{B_{d B}}{10}}=10^{X_{d B}}=e^{\Lambda_{1}}+e^{\Lambda_{2}}$. Therefore, $\Lambda_{1}=\rho \times A_{(d B)}$ and $\Lambda_{2}=\rho \times B_{(d B)}$. The relevant correlation coefficient of $A$ and $B$ can be given as

$$
r_{A, B}=\frac{\operatorname{cov}\left(A_{(d B)}, B_{(d B)}\right)}{\sqrt{\operatorname{var}\left(A_{(d B)}\right) \operatorname{var}\left(B_{(d B)}\right)}}=\frac{\sigma_{S}}{\sqrt{\sigma_{S}^{2}+\sigma_{V}^{2}}}
$$

where $\sigma_{S}$ and $\sigma_{V}$ can be calculated based on the DTT transmitter information used for geo-location database calculation algorithm. Based on (22) and (23), $\mu_{1}$ and $\mu_{2}$ can be obtained. Consequently, $m_{X}$ and $\sigma_{X}$ can be calculated according to (24) and (25).

Similarly, $q_{2}$ can be calculated by Wilkinson's method by the following procedure:

1) Input $m_{S}, \sigma_{S}, m_{V}, \sigma_{V}, m_{C}$ and $\sigma_{C}$ as shown in (10), which can be calculated based on the DTT transmitter information used for geo-location database calculation algorithm;
2) Calculate $m_{D}$ and $\sigma_{D}$ by Wilkinson's method based on $m_{V}, \sigma_{V}, m_{C}$ and $\sigma_{C}$

3) Calculate $m_{A}, \sigma_{A}, m_{E}$ and $\sigma_{E}$ by Wilkinson's method based on $m_{S}, \sigma_{S}, m_{D}$ and $\sigma_{D}$;

4) Calculate $m_{Y}$ and $\sigma_{Y}$ by Wilkinson's method based on $m_{A}, \sigma_{A}, m_{E}$ and $\sigma_{E}$

5) Calculate $q_{2}$ by (11) based on $m_{A}, \sigma_{A}, m_{E}$ and $\sigma_{E}$.

The procedure of calculating $P_{I B}$ with the Wilkinson's method is shown as Fig. 2 and summarized as follows. Firstly, input the mean and standard derivation of the received power of wanted DTT signal, i.e., $P_{s}$ and the minimum required power of wanted DTT signal, i.e., $V$, which can be obtained from the DTT transmitter information used for geo-location database calculation algorithm. As defined in IEEE 802.22 standard, the maximum allowable EIRP that can be utilized in TV frequency band is 4 watts [47]. Therefore, the predefined maximum allowable value $\left(4\right.$ watts) is assigned to $P_{I B}$ for each TVWS channel. In addition, $q_{1}$ and $q_{2}$ are calculated by the Wilkinson's method as aforementioned. Consequently, the corresponding $P_{I B}$ is updated until $q_{2} \leq q_{1}-\Delta q_{T}$.

\section{NumericAl ANALYSES}

The analyses of the proposed stand-alone hybrid framework on the simulated signals and data are presented in this section. Furthermore, the proposed hybrid framework is tested on the real-time signals collected by RFeye node and the data obtained from the geo-location database provided by Nominet.

\section{A. Numerical analyses on simulated signals and data}

In the simulations, orthogonal frequency-division multiplexing (OFDM) signals are simulated as PUs, which is used by the DVB-T signals in TVWS from $470 \mathrm{MHz}$ to $790 \mathrm{MHz}$ in the UK [2]. There are a total of 40 channels in TVWS with a bandwidth of $8 \mathrm{MHz}$ for each channel. It is assumed that each PU is independent and only locates at one channel. The transmission channel for signals is modeled as an AWGN channel. In addition, the signal-to-noise ratio (SNR) is defined as the ration of received signal power to the noise power in each TVWS channel. The target $P_{f}$ is set to be 0.01 .

The comparison of the proposed and traditional methods for calculating maximum allowable EIRP are presented firstly. Since Monte Carlo simulation is based on no assumption and approximation, its results can be considered precise as long as the number of trials is large enough. With 10,000 points, Monte Carlo simulation shows a relatively stable performance. By taking the results obtained by Monte Carlo simulation as a benchmark, the accuracy of the Schwartz-Yeh's method and Wilkinson's method can be measured by error rate $\Delta Q(\cdot) / Q_{\text {(MonteCarlo) }}(\cdot)$, where $Q_{\text {(MonteCarlo) }}(\cdot)$ refers to values calculated by Monte Carlo simulation and $\Delta Q(\cdot)$ refers to the absolute difference of parameters' values between Schwartz-Yeh's method or Wilkinson's method and the Monte Carlo simulation. More specifically, $\Delta Q\left(q_{1}\right)=\left|q_{1}^{S, W}-q_{1}^{M}\right|$, $\Delta Q\left(q_{2}\right)=\left|q_{2}^{S, W}-q_{2}^{M}\right|$ and $\Delta Q\left(P_{I B}\right)=\left|P_{I B}^{S, W}-P_{I B}^{M}\right|$, where $q_{1}^{S, W}, q_{2}^{S, W}$ and $P_{I B}^{S, W}$ refer to the corresponding values calculated by the Schwartz-Yeh's method or Wilkinson's 


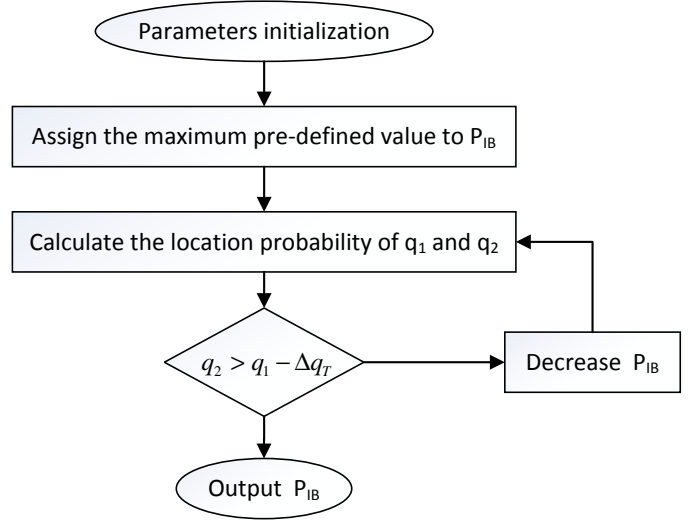

Fig. 2: The procedure of calculating maximum allowable $P_{I B}$ by the Wilkinson's method.

method respectively, and $q_{1}^{M}, q_{2}^{M}$ and $P_{I B}^{M}$ refer to the corresponding values calculated by Monte Carlo simulation. The error rates of $q_{1}, q_{2}$ and $P_{I B}$ calculated by the Schwartz-Yeh's method and Wilkinson's method are shown in Table I. We can see that the proposed Wilkinson's method outperforms the Schwartz-Yeh's method in terms of the calculation accuracy.

Similarly as the error rate calculation, running time of Monte Carlo simulation with 10,000 points is chosen as a benchmark when measuring the running time for the calculation of $q_{1}, q_{2}$ and $q_{I B}$. Table II shows the running time comparison of the Schwartz-Yeh's and Wilkinson's methods. We can see that the Wilkinson's method reduces the running time significantly in comparison with the Schwartz-Yeh's method. Therefore, the proposed Wilkinson's method is very suitable for SUs with limited power to obtain the $q_{1}, q_{2}$ and $P_{I B}$ efficiently.

After validating the accuracy and efficiency, a national grid reference (NGR) based geo-location database is built with the proposed Wilkinson's method. By utilizing the DTT transmitter location information for geo-location database calculation algorithm, $P_{I B}$ can be calculated by the proposed Wilkinson's method based DTT location probability model for any specific location. We choose an NGR number of SP515065 in Oxford as a test location. The maximum allowable EIRP calculated by the power control and the proposed location probability model are shown in Table III.

As shown in Table III, there are 11 available channels at SP515065 in total. In the proposed location probability model, the transmission environment is classified into three situations: open, suburban and urban. Coupling gain in different situations is treated differently, leading to different interference toleration levels of DTT receivers. It is obvious that the power attenuation in open areas is much lower than suburban and urban areas. As a result, the actual maximum allowable EIRP $P_{I B}$ in open areas is smaller than the other two situations at a certain NGR location. Taking channel 51 as an example, the $P_{I B}$ is 0.0002 watts in power control model. However, the spectrum of interest could be utilized more effectively if the transmission environment is classified, which is 0.3981 watts in open areas, 1.2589 watts in suburban areas and 4.0000 watts in urban areas.
TABLE I: Error rates comparison

\begin{tabular}{|c|c|c|c|}
\hline & $q_{1}$ & $q_{2}$ & $P_{I B}$ \\
\hline Schwartz-Yeh's method & $31.25 \%$ & $4.76 \%$ & $7.87 \%$ \\
\hline Wilkinson's method & $9.36 \%$ & $1.31 \%$ & $1.54 \%$ \\
\hline
\end{tabular}

TABLE II: Running time comparison.

\begin{tabular}{|c|c|c|c|}
\hline & $q_{1}$ & $q_{2}$ & $P_{I B}$ \\
\hline Schwartz-Yeh's method & $15966.04 \%$ & $153278.65 \%$ & $75462.57 \%$ \\
\hline Wilkinson's method & $99.06 \%$ & $98.89 \%$ & $99.47 \%$ \\
\hline
\end{tabular}

TABLE III: Comparison of actual maximum allowable EIRP $P_{I B}$ in Oxford.

\begin{tabular}{|c|c|c|c|c|}
\hline \multirow{2}{*}{} & \multicolumn{3}{|c|}{ Actual Maximum Allowable EIRP $P_{I B}$ (Watt) } \\
\cline { 2 - 4 } & \multicolumn{2}{|c|}{$\begin{array}{c}\text { The latest release of Ofcom TV white } \\
\text { space model by Wilkinson's method }\end{array}$} & \multirow{2}{*}{$\begin{array}{c}\text { Power } \\
\text { control model }\end{array}$} \\
\hline $\begin{array}{c}\text { Available } \\
\text { Channel }\end{array}$ & Open & Suburban & Urban & 4.0000 \\
\hline 22 & 0 & 4.0000 & 4.0000 & 4.0000 \\
\hline 25 & 0 & 4.0000 & 4.0000 & 4.0000 \\
\hline 28 & 0 & 4.0000 & 4.0000 & 4.0000 \\
\hline 29 & 0.0025 & 4.0000 & 4.0000 & 4.0000 \\
\hline 40 & 0 & 4.0000 & 4.0000 & 4.0000 \\
\hline 43 & 0 & 4.0000 & 4.0000 & 4.0000 \\
\hline 46 & 0 & 4.0000 & 4.0000 & 0.0002 \\
\hline 49 & 0.0013 & 4.0000 & 4.0000 & 4.0000 \\
\hline 51 & 0.3981 & 1.2589 & 4.0000 & 4.0000 \\
\hline 54 & 0.0013 & 4.0000 & 4.0000 & \\
\hline 58 & 0.0013 & 4.0000 & 4.0000 & \\
\hline
\end{tabular}

Based on the obtained $P_{I B}$ from the local geo-location database algorithm, the weights are constructed by fusing the current $P_{I B}$ with historical data in the proposed DNRLS based compressive spectrum sensing. Fig. 3 shows detection performance of the sensing only approach and the proposed hybrid framework with DNRLS algorithms implemented at SUs, where $p$ is set to be 0.1. It is observed that the detection performance of the sensing only approach without CS implemented at a SU is matched with the theoretical curve, which is presented as a benchmark and can be expressed as

$$
P_{d}=Q\left(\frac{\frac{\lambda_{d}}{\sigma^{2}}-\left(1+\frac{\sigma_{s}^{2}}{\sigma^{2}}\right)}{\left(1+\frac{\sigma_{s}^{2}}{\sigma^{2}}\right) / \sqrt{\frac{N}{2}}}\right),
$$

where $\lambda_{d}$ is the threshold for energy detection as calculated by (5) and $P_{d}$ refers to detection probability of spectrum sensing system.

Fig. 3 shows that detection performance of the sensing only approach with IRLS is smaller than the theoretic curve due to the signal recovery errors caused by the sub-Nyquist sampling (20\%). When the proposed hybrid framework with DNRLS is performed, detection probability increases greatly which can almost match with the theoretic curve. The reason for the large performance improvement is that the data used to construct the weights is the exact representation of the spectrum of interest if there is no unregistered user. In addition, it is noted that the sensing only approach with IRLS requires an iterative process to update the weights. This iterative process introduces a higher computational complexity. As a result, the proposed DNRLS based compressive spectrum sensing can achieve better detection performance with $(L-1) / L$ of 


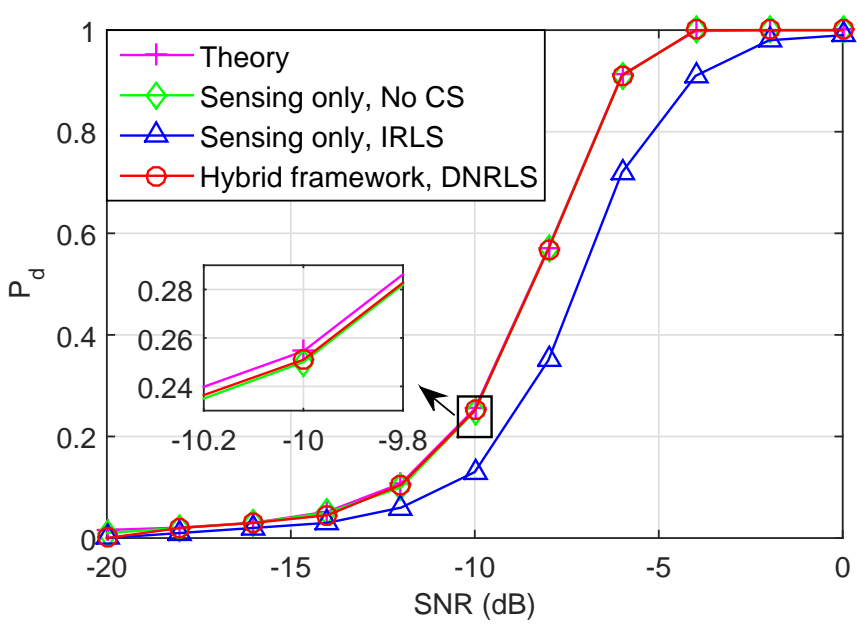

Fig. 3: Detection performance on the simulated signals and data under different SNR values, $p=0.1$, compression ratio $=20 \%$.

computational complexity reduced in comparison with the sensing only approach with IRLS.

Fig. 4 shows detection probability of the sensing only approach with IRLS and the proposed hybrid framework with DNRLS with varying compression ratios. In this scenario, the spectrum occupancy ratio is assumed to be $12.5 \%, p$ is 0.1 and the SNR value is $-5 \mathrm{~dB}$. It is noted that there is a big difference on the necessary number of measurements between the proposed hybrid framework and the sensing only approach to achieve the same detection probability. Specifically, as shown in Fig. 4, the proposed hybrid framework with DNRLS can achieve $90 \%$ detection probability when the compression ratio is no higher than $7 \%$. However, the sensing only approach requires the compression ratio to be about $20 \%$ in order to achieve the same performance. As a result, the sampling rates can be reduced by $13 \%$ without degrading the detection performance.

The detection performance of the proposed hybrid framework with DNRLS is shown in Fig. 5 with different spectrum occupancy ratios in TVWS and different $p$ values for $l_{p}$. In this scenario, SNR is set to be $-5 \mathrm{~dB}$ and the positions of these active PUs are set to be random. In compressive spectrum sensing, increasing spectrum occupancy in spectrum of interest refers to higher sparsity levels of the signal to be recovered. We can see that the detection performance becomes improved with decreasing value of $p$ and fixed sparsity level. Meanwhile, the detection performance is degraded slightly with increasing sparsity level increases when the value for $p$ is fixed. As a result, more compressed measurements should be collected at SUs to avoid performance degradation when sparsity level increases.

Fig. 6 shows the detection probability of the proposed hybrid framework with DNRLS under different window sizes $T$ with new unregistered users showing up in the spectrum of interest. In this scenario, the spectrum occupancy is $12.5 \%, p$ is 0.1 and compression ratio is $10 \%$. With unregistered users in TVWS, only half of the weights for the channels with active

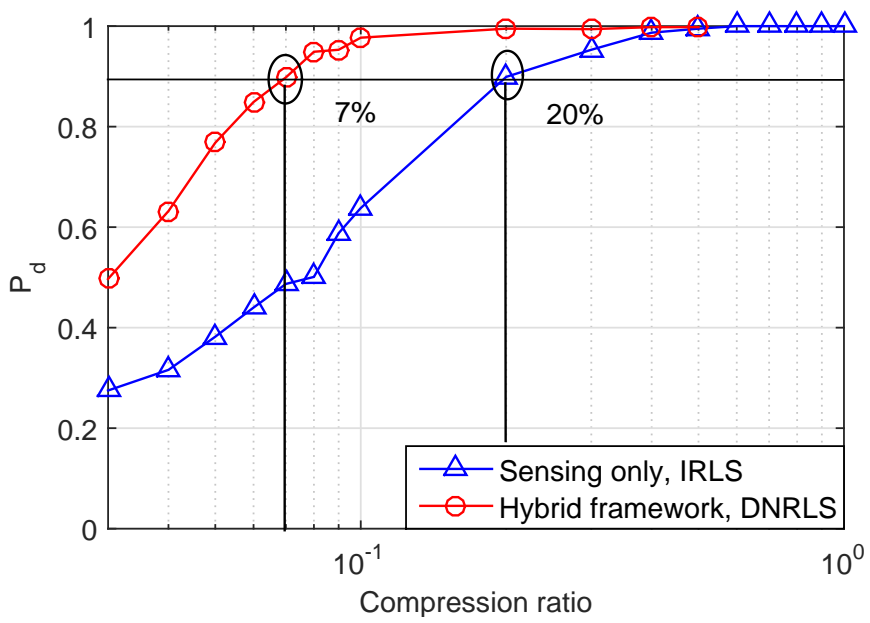

Fig. 4: Detection performance on the simulated signals and data under different compression ratios, $p=0.1, \mathrm{SNR}=-5 \mathrm{~dB}$.

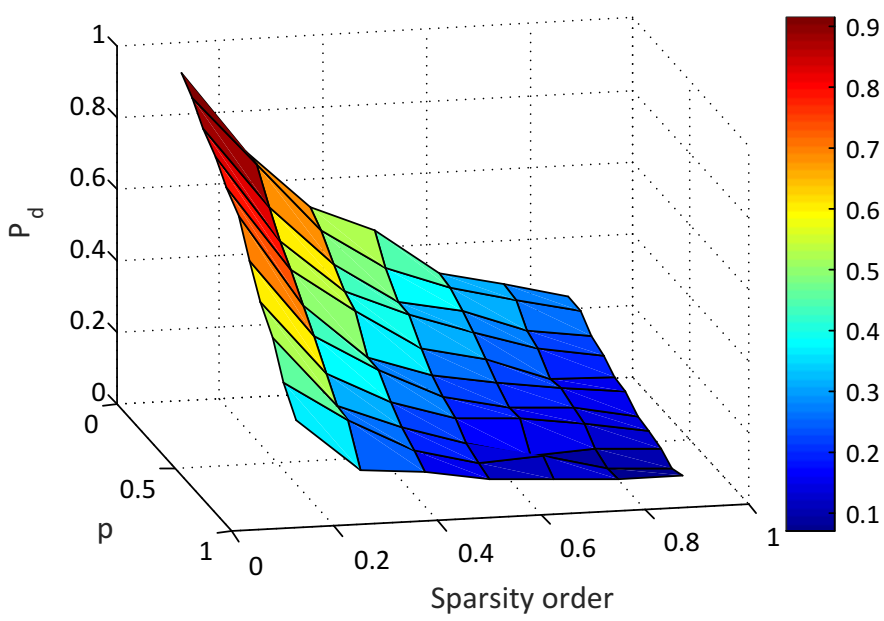

Fig. 5: Detection performance on the simulated signals and data under different sparsity levels and $p$ values, compression ratio $=10 \%, \mathrm{SNR}=-5 \mathrm{~dB}$.

PUs are exact. We can see that the detection performance is degraded from $98 \%$ to $85 \%$ in the first sensing period after a new unregistered user shows up in TVWS. However, after one sensing period passed, which refers to $T=2$, the detection performance is improved to about $95 \%$. This improvement benefits from the weights are constructed by fusing the output of the geo-location database algorithm with the historical data. The geo-location database algorithm utilizes the selfmaintained geo-location database at SU locally which contains the new unregistered users' information. Furthermore, the detection performance converges to $98 \%$ after four updates of the weights. With increasing window size $T$, the improvement on detection performance becomes slower after the first updating on the weights. However, if the unregistered user shows up again in the same position of TVWS, detection probability of the proposed hybrid framework with DNRLS falls between $85 \%$ and $95 \%$, which is dependent on the window size $T$. If $T$ is large enough, the detection probability would get close to $95 \%$. 


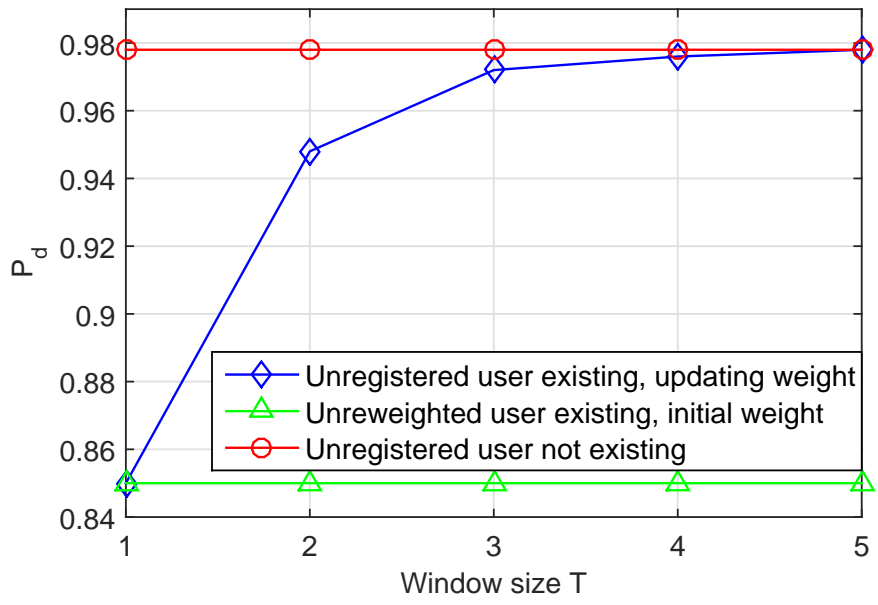

Fig. 6: Detection performance on the simulated signals and data under different window sizes $T$ with unregistered users existing, compression ratio $=10 \%, p=0.1, \mathrm{SNR}=-5 \mathrm{~dB}$.

\section{B. Numerical analyses on real-time signals and data}

After the proposed DNRLS compressive spectrum sensing algorithm is validated by the simulated signals and data, the proposed framework is tested on real-time signals collected by the CRFS RFeye node and the real data provided by the geo-location database from Nominet qualified by Ofcom. The RFeye node is scalable and cost-effective which can provide real-time 24/7 monitoring of the radio spectrum. It is capable of sweeping spectrum from $10 \mathrm{MHz}$ to $6 \mathrm{GHz}$, and capture signals of all types, including transient transmission such as pulsing or short-burst signals. It is located at Queen Mary University of London $\left(51.523021^{\circ} \mathrm{N} 0.041592^{\circ} \mathrm{W}\right)$, and the antenna height is about 15 meters above ground.

Some pilots in TVWS have been undertaken in the UK as launched by the Ofcom. In part the trails run at QMUL, an unregistered user is transmitted in TVWS channel 27 $(518 \mathrm{MHz}$ to $526 \mathrm{MHz})$. In this case, the historical data and $P_{I B}$ from the geo-location database would not be exact for the channel 27 as it is the first time for the unregistered user showing up in TVWS. As a result, the output of the geolocation database would still allow a high EIRP in channel 27. The simulation results for the case with unregistered users under different window sizes $T$ are shown in Fig. 7. We can see that the detection performance would be degraded once the unregistered user shows up in TVWS. This is caused by the inexact weights constructed by the inaccurate $P_{I B}$ in channel 27. Similarly as Fig. 6, the detection performance is increased largely after window size $T$ is increased to 2 . With increasing window size, the detection performance of the proposed hybrid framework with DNRLS converges efficiently.

Based on the fast convergence performance shown in Fig. 6 and Fig. 7, we can reason out the practicability of our proposed hybrid framework is reasonable. The implementation of compressive spectrum sensing with a geo-location database algorithm can improve the energy efficiency at SUs by reducing its computational complexities. Therefore, such an energy efficient algorithm could be applied to multiple scenarios with

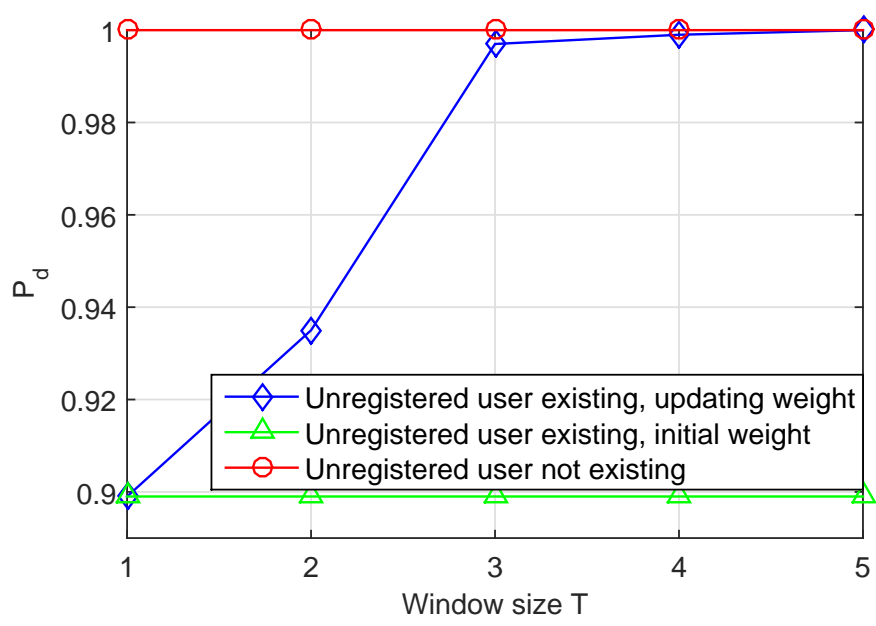

Fig. 7: Detection performance on the real-time signals under different window sizes $T$ with unregistered users existing, compression ratio $=10 \%, p=0.1$.

power-limited WSDs in a M2M communication network.

\section{CONCLUSIONS}

A stand-alone hybrid framework combining compressive spectrum sensing and geo-location database was designed for wideband spectrum in this paper. In particular, a data-assisted non-iteratively reweighted least squares (DNRLS) based compressive spectrum sensing algorithm was proposed to reduce the sampling rates to under sub-Nyquist rate and lower the computational complexities by exploiting the data provided by the geo-location database algorithm stored at secondary users (SUs) locally. Furthermore, based on the recent trail within the Ofcom TV white space pilot, the proposed framework was tested on the real-time signals and data after having been validated by the simulated signals and data in TVWS. The numerical results showed that the computational complexities of signal recovery process were reduced by $(L-1) / L$ in terms of number of iterations, and the detection performance was improved by implementing the proposed DNRLS algorithm in comparison with traditional algorithms. This makes it possible to apply the proposed framework to energy-constraint terminals, such as Machine-to-Machine (M2M) sensors.

The proposed hybrid framework can also provide benefits to relax the requirement on sparsity level estimation in compressive spectrum sensing. More specifically, the compression ratio at SUs is difficult to determine in existing compressive sensing algorithms, as the sparsity level is unknown before the compressive spectrum sensing is performed. In our proposed hybrid framework, the data from geo-location database calculation algorithm can be used to provide an estimation of the sparsity level. As a result, the lowest compression ratio guaranteing exact recovery can be determined by the wellknown relationship between necessary measurements and the sparsity level.

Besides on the aforementioned advantages, the proposed framework also has disadvantages. In the implementation of a 
locally stored geo-location database algorithm, extra computational complexities would be introduced at SUs even though an efficient geo-location database algorithm has been proposed in this paper. It is noted from our live spectrum monitoring system that the spectrum may not change in a period of time, which means the output of geo-location database algorithm may not change frequently. Therefore, how often should the geo-location database algorithm be called at SUs is an issue to be analyzed with respect to the variations of spectrum. This could be achieved by further theoretical analysis with more experiments on the real-time spectrum data in the future. With these further improvements and more experiments, the proposed hybrid framework can be extended to much wider bands by including spectrum activities in cellular, industrial, scientific and medical (ISM) frequency bands.

\section{ACKNOWLEDGEMENT}

The authors would like to acknowledge the Engineering and Physical Sciences Research Council (EPSRC) in the UK for their support of this work with Grant No. EP/L024241/1. The authors would also like to thank Nominet and Ofcom for providing access to the geo-location database, and CRFS Ltd for providing the RFeye node.

\section{REFERENCES}

[1] P. Kolodzy and I. Avoidance, "Spectrum policy task force," Federal Commun. Comm., Washington, DC, Rep. ET Docket, no. 02-135, 2002.

[2] UK Office of Communications (Ofcom), Statement on Cognitive Access to Interleaved Spectrum, Jul. 2009.

[3] I. Mitola, J. and J. Maguire, G.Q., "Cognitive radio: making software radios more personal,' IEEE IEEE Pers. Commun., vol. 6, no. 4, pp. 13 -18 , Aug. 1999.

[4] Federal Communications Commission (FCC), FCC Frees Up Vacant TV Airwaves for Super Wi-Fi, Technologies and Other Technologies, 2010. [Online]. Available: https://apps.fcc.gov/edocs_ public/attachmatch/DOC-301650A1.pdf

[5] UK Office of Communications (Ofcom), Ofcom Implementing TV White Spaces, Feb. 2015. [Online]. Available: http://stakeholders.ofcom.org.uk/binaries/consultations/ white-space-coexistence/statement/tvws-statement.pdf

[6] F. Paisana, N. Marchetti, and L. DaSilva, "Radar, TV and cellular bands: Which spectrum access techniques for which bands?" IEEE Commun. Surveys Tutorials, vol. 16, no. 3, pp. 1193-1220, Third Quarter 2014.

[7] J. Ribeiro, J. Ribeiro, J. Rodriguez, R. Dionisio, H. Esteves, P. Duarte, and P. Marques, "Testbed for combination of local sensing with geolocation database in real environments," IEEE Wireless Commun., vol. 19, no. 4, pp. 59-66, Aug. 2012.

[8] J. Wang, G. Ding, Q. Wu, L. Shen, and F. Song, "Spatial-temporal spectrum hole discovery: a hybrid spectrum sensing and geolocation database framework," Chinese Science Bulletin, vol. 59, no. 16, pp. 1896-1902, 2014.

[9] N. Wang, Y. Gao, and B. Evans, "Database-augmented spectrum sensing algorithm for cognitive radio," in Proc. IEEE Int. Conf. Commun. (ICC), London, UK, Jun. 2015.

[10] Z. Qin, L. Wei, Y. Gao, and C. Parini, "Compressive spectrum sensing augmented by geo-location database," in International Workshop on Smart Spectrum at IEEE Wireless Communication and Networking Conference (WCNC), New Orleans, LA, Mar. 2015, pp. 1170-1174.

[11] Z. Quan, S. Cui, A. Sayed, and H. Poor, "Optimal multiband joint detection for spectrum sensing in cognitive radio networks," IEEE Trans. Signal Process., vol. 57, no. 3, pp. 1128-1140, Mar. 2009.

[12] Z. Tian and G. B. Giannakis, "A wavelet approach to wideband spectrum sensing for cognitive radios," in 1st International Conference on Cognitive Radio Oriented Wireless Networks and Communications (CROWNCOM), Mykonos Island, Greece, June 2006, pp. 1 -5.

[13] R. Zhang and C. K. Ho, "MIMO broadcasting for simultaneous wireless information and power transfer," IEEE Trans. Commun., vol. 12, no. 5, pp. 1989-2001, Mar. 2013.
[14] H. Landau, "Necessary density conditions for sampling and interpolation of certain entire functions," Acta Mathematica, vol. 117, no. 1, pp. 37$52,1967$.

[15] E. Candes, "Compressive sampling," in Proceedings of the International Congress of Mathematicians, vol. 3, Madrid, Spain, 2006, pp. 14331452 .

[16] Z. Tian and G. Giannakis, "Compressed sensing for wideband cognitive radios," in IEEE International Conference on Acoustics, Speech and Signal Processing (ICASSP), Honolulu, HI, Apr. 2007, pp. 1357-1360.

[17] Y. Wang, Z. Tian, and C. Feng, "Sparsity order estimation and its application in compressive spectrum sensing for cognitive radios," IEEE Trans. Wireless Commun., vol. 11, no. 6, pp. 2116-2125, 2012.

[18] H. Sun, W.-Y. Chiu, and A. Nallanathan, "Adaptive compressive spectrum sensing for wideband cognitive radios," IEEE Commun. Lett., vol. 16, no. 11, pp. 1812-1815, Nov. 2012.

[19] Z. Qin, Y. Gao, M. Plumbley, and C. Parini, "Efficient compressive spectrum sensing algorithm for M2M devices," in IEEE Global Conference on Signal and Information Processing (GlobalSIP), Atlanta, GA, Dec 2014, pp. 1170-1174.

[20] M. Mishali and Y. Eldar, "From theory to practice: Sub-nyquist sampling of sparse wideband analog signals," IEEE J. Sel. Signal Process., vol. 4, no. 2, pp. 375-391, Apr. 2010.

[21] J. Tropp, J. Laska, M. Duarte, J. Romberg, and R. Baraniuk, "Beyond nyquist: Efficient sampling of sparse bandlimited signals," IEEE Trans. Inf. Theory, vol. 56, no. 1, pp. 520-544, Jan. 2010.

[22] E. J. Candes, M. B. Wakin, and S. P. Boyd, "Enhancing sparsity by reweighted 11 minimization," Journal of Fourier Analysis and Applications, vol. 14, no. 5-6, pp. 877-905, 2008.

[23] R. Chartrand, "Exact reconstruction of sparse signals via nonconvex minimization," IEEE Signal Process. Lett., vol. 14, no. 10, pp. 707710, Oct. 2007.

[24] R. Chartrand and W. Yin, "Iteratively reweighted algorithms for compressive sensing," in IEEE International Conference on Acoustics, Speech and Signal Processing (ICASSP), Las Vegas, NV, Mar. 2008, pp. 3869-3872.

[25] R. Chartrand and V. Staneva, "Restricted isometry properties and nonconvex compressive sensing," Inverse Problems, vol. 24, no. 3, p. 035020, 2008.

[26] R. E. Carrillo and K. Barner, "Iteratively re-weighted least squares for sparse signal reconstruction from noisy measurements," in 43rd Annual Conference on Information Sciences and Systems (CISS), Baltimore, MD, Mar. 2009, pp. 448-453.

[27] R. Saab and Ö. Y1lmaz, "Sparse recovery by non-convex optimizationinstance optimality," Applied and Computational Harmonic Analysis, vol. 29, no. 1, pp. 30-48, 2010.

[28] D. Ba, B. Babadi, P. Purdon, and E. Brown, "Convergence and stability of iteratively re-weighted least squares algorithms," IEEE Trans. Signal Process., vol. 62, no. 1, pp. 183-195, Jan. 2014.

[29] O. Escoda, L. Granai, and P. Vandergheynst, "On the use of a priori information for sparse signal approximations," IEEE Trans. Signal Process., vol. 54, no. 9, pp. 3468-3482, Sep. 2006.

[30] M. Friedlander, H. Mansour, R. Saab, and O. Yilmaz, "Recovering compressively sampled signals using partial support information," IEEE Trans. Inf. Theory, vol. 58, no. 2, pp. 1122-1134, Feb. 2012.

[31] W. Lu and N. Vaswani, "Regularized modified bpdn for noisy sparse reconstruction with partial erroneous support and signal value knowledge," IEEE Trans. Signal Process., vol. 60, no. 1, pp. 182-196, 2012.

[32] C. J. Miosso, R. von Borries, M. Argaez, L. Velázquez, C. Quintero, and C. Potes, "Compressive sensing reconstruction with prior information by iteratively reweighted least-squares," IEEE Trans. Signal Process., vol. 57, no. 6, pp. 2424-2431, 2009.

[33] D. Wipf and S. Nagarajan, "Iterative reweighted 11 and 12 methods for finding sparse solutions," IEEE J. Sel. Signal Process., vol. 4, no. 2, pp. 317-329, Apr. 2010.

[34] O. Holland, S. Ping, Y. Gao, Z. Qin, A. Aijaz, J. Chareau, P. Chawdhry, and H. Kokkinen, "To white space or not to white space: That is the trial within the ofcom TV white spaces pilot," in IEEE International Symposium on Dynamic Spectrum Access Networks (DYSPAN), Stockholm, Sweden, Sep. 2015, (accepted).

[35] RFeye Node. [Online]. Available: http://www.crfs.com/products/ rf-sensor-rfeye-node/

[36] UK Office of Communications (Ofcom), TV white spaces: approach to coexistece. [Online]. Available: http://stakeholders.ofcom.org.uk/ spectrum/tv-white-spaces/databases/

[37] J. Treichler, M. Davenport, and R. Baraniuk, "Application of compressive sensing to the design of wideband signal acquisition receivers," 
US/Australia Joint Work. Defense Apps. of Signal Processing (DASP), Lihue, Hawaii, vol. 5, Sep. 2009.

[38] Z. Ye, G. Memik, and J. Grosspietsch, "Energy detection using estimated noise variance for spectrum sensing in cognitive radio networks," in IEEE Wireless Communications and Networking Conference (WCNC), Las Vegas, NV, Mar. 2008, pp. 711-716.

[39] S. Gong, W. Liu, W. Yuan, W. Cheng, and S. Wang, "Threshold-learning in local spectrum sensing of cognitive radio," in IEEE 69th Vehicular Technology Conference, (VTC Spring), Barcelona, Spain, Apr. 2009, pp. $1-6$.

[40] M. Bkassiny, S. Jayaweera, Y. Li, and K. Avery, "Wideband spectrum sensing and non-parametric signal classification for autonomous selflearning cognitive radios," IEEE Trans. Wireless Commun., vol. 11, no. 7, pp. 2596-2605, Jul. 2012.

[41] D. Joshi, D. Popescu, and O. Dobre, "Adaptive spectrum sensing with noise variance estimation for dynamic cognitive radio systems," in 44th Annual Conference on Information Sciences and Systems (CISS), Princeton, NJ, Mar. 2010, pp. 1-5.

[42] N. Wang, Y. Gao, Y. Chen, E. Bodanese, and L. Cuthbert, "A power control algorithm for TV white space cognitive radio system," in IET International Conference on Communication Technology and Application (ICCTA), London, UK, Oct. 2011, pp. 546-550.

[43] UK Office of Communications (Ofcom), TV white spaces- approach to coexistence, p. 26, Sep, 2013. [Online]. Available: http://stakeholders. ofcom.org.uk/consultations/white-space-coexistence/

[44] S. C. Schwartz and Y.-S. Yeh, "On the distribution function and moments of power sums with log-normal components," Bell System Technical Journal, vol. 61, no. 7, pp. 1441-1462, 1982.

[45] L. Fenton, "The sum of log-normal probability distributions in scatter transmission systems," IRE Transactions on Communications Systems, vol. 8, no. 1, pp. 57-67, Mar. 1960.

[46] V. Petrini and H. Karimi, "TV white space databases: algorithms for the calculation of maximum permitted radiated power levels," in IEEE International Symposium on Dynamic Spectrum Access Networks (DYSPAN), Bellevue, WA, Oct 2012, pp. 552-560.

[47] "IEEE Standard for Wireless Regional Area Networks Part 22: Cognitive Wireless RAN MAC \& PHY Specifications: Policies and Procedures for Operation in the TV Bands," IEEE Std. 802.22-2011, 2011. 